\title{
Frequency-dependent data weighting in global gravity field modeling from satellite data contaminated by non-stationary noise
}

Received: 14 July 2005 / Accepted: 16 June 2006 / Published online: 21 July 2006

(C) Springer-Verlag 2006

\begin{abstract}
Satellite data that are used to model the global gravity field of the Earth are typically corrupted by correlated noise, which can be related to a frequency dependence of the data accuracy. We show an opportunity to take such noise into account by using a proper noise covariance matrix in the estimation procedure. If the dependence of noise on frequency is not known a priori, it can be estimated on the basis of a posteriori residuals. The methodology can be applied to data with gaps. Non-stationarity of noise can also be dealt with, provided that the necessary a priori information exists. The proposed methodology is illustrated with CHAllenging Minisatellite Payload (CHAMP) data processing. It is shown, in particular, that the usage of a proper noise model can make the measurements of non-gravitational satellite accelerations unnecessarily. This opens the door for high-quality modeling of the Earth's gravity field on the basis of observed orbits of non-dedicated satellites (i.e., satellites without an on-board accelerometer). Furthermore, the processing of data from dedicated satellite missions - GRACE (Gravity Recovery and Climate Experiment) and GOCE (Gravity field and steady-state Ocean Circulation Explorer) - may also benefit from the proposed methodology.
\end{abstract}

Keywords Earth's gravity field - Colored noise - Covariance matrix estimation - CHAMP (CHAllenging Mini-satellite Payload) $\cdot$ Satellite-borne accelerometer

P. Ditmar $(\varangle) \cdot$ R. Klees $\cdot$ X. Liu

Delft Institute of Earth Observation and Space Systems (DEOS),

Faculty of Aerospace Engineering,

Delft University of Technology,

Kluyverweg 1, 2629 HS Delft, The Netherlands

E-mail: p.ditmar@lr.tudelft.nl

Tel.: +31-15-2782501

Fax: +31-15-2783711

E-mail: r.klees@1r.tudelft.nl

E-mail: x.1.liu@lr.tudelft.nl

\section{Introduction}

Thanks to new dedicated satellite gravimetry missions, our knowledge of the Earth's gravity field has significantly improved in recent years. The CHAMP (CHAllenging Minisatellite Payload) mission (Reigber et al. 1996) has resulted in models of 20-cm accuracy in terms of geoid heights up to spherical harmonic degree and order 70 (Reigber et al. 2005a; Wermuth et al. 2004; Mayer-Gürr et al. 2005; Ditmar et al. 2006b). The improvement with respect to earlier models, e.g. EGM96 (Lemoine et al. 1998), is especially noticeable in geographically remote areas, where terrestrial measurements are (or were) not available.

The successive GRACE (Gravity Recovery and Climate Experiment) satellite mission (Tapley 1997) allowed the accuracy of gravity field models to be increased further to a $3-\mathrm{cm}$ geoid height error for a model complete to degree and order 100 (Reigber et al. 2005b; Förste et al. 2005; Tapley et al. 2005). Furthermore, GRACE is the first satellite mission ever that could observe temporal gravity field variations at a regional scale (Tapley et al. 2004; Wahr et al. 2004).

It is expected that the upcoming GOCE (Gravity field and steady-state Ocean Circulation Explorer) satellite mission (ESA 1999) will increase the accuracy of global gravity field models to $1 \mathrm{~cm}$ up to degree 200 (ESA 1999; Bouman 2000; Sneeuw 2002; Ditmar et al. 2003).

A crucial aspect of high-precision gravity field modeling is a proper data weighting, which requires an adequate noise model. Such a model, in particular, may include a dependence of noise on frequency. A number of different concepts has been proposed on how to deal with colored (i.e. frequency dependent) noise in satellite gravimetry data. Migliaccio et al. (2004) proposed to apply a Wiener filter to the data. Since such a filter influences both noise and signal, a number of iterations are needed until the computed gravity field model matches the observations. Alternatively, Schuh (1996) proposed a decorrelation of the functional model with autoregressive moving average (ARMA) filters. Later, this idea was realized in efficient computational algorithms suitable 
for solving huge systems of linear equations (Schuh 2003; Klees et al. 2003). Furthermore, Klees and Broersen (2002) and Klees et al. (2003) presented a statistically justified methodology to derive ARMA-filter coefficients from a noise power spectral density (PSD) or directly from a noise realization.

A potential pitfall of decorrelation by filtering is edge effects, which may show up not only at the beginning and at the end of the entire data series but also in the vicinity of each data gap. According to Schuh (2003), this problem can be solved by removing from the decorrelated functional model a few epochs after each gap ("filter warming-up phase"). The price to pay is an unnecessary loss of data, which can be significant in the presence of multiple gaps. Another approach is building up the noise covariance matrix explicitly (Reubelt et al. 2003), which may of course be time consuming in the case of a large data set.

An alternative solution to the problem of data gaps is a low-level pre-conditioned conjugate gradient (PCCG) algorithm based on ARMA filters, which does not introduce any approximations in dealing with the noise covariance matrix, even in the presence of gaps (Klees and Ditmar 2004). Furthermore, Ditmar and van Eck van der Sluijs (2004) proposed a modified technique for numerically efficient data weighting by means of the low-level PCCG scheme; the pre-requisite is that the noise dependence on frequency can be approximated by a certain analytic relationship. Ditmar et al. (2004b) showed how this procedure can take non-stationary noise into account. Ditmar et al. (2006b) exploited the proposed data weighting scheme to produce the global gravity field model DEOS_CHAMP-01C_70, which can compete with the best CHAMP-based models produced by other methods and other groups. Ditmar et al. (2006a) discussed how to define parameters of the analytic noise representation from the data themselves.

In the current publication, we summarize our previous experience and generalize it yet further. It is now possible to consider any dependence of noise on frequency in combination with non-stationary noise. Furthermore, we discuss how to determine the dependence of noise on frequency from the data. The presented methodology is applied to CHAMP accelerations derived from a kinematic satellite orbit. The data are processed according to two different scenarios: (i) the observed satellite accelerations are cleaned from non-gravitational accelerations, which are measured by the on-board accelerometer; and (ii) the non-gravitational accelerations are completely ignored. The second scenario allows one to assess the accuracy of gravity field modeling that can be expected if data from a non-dedicated satellite (i.e. a satellite without an accelerometer) are used.

The structure of the paper is as follows: Section 2 presents the general principles of gravity field modeling on the basis of satellite data. Section 3 discusses a noise model and a way to derive its parameters from the data. Section $4 \mathrm{dem}-$ onstrates how the presented methodology can be applied to real satellite data. Section 5 contains a discussion and some conclusions.

\section{Gravity field modeling}

The most traditional representation of the global gravity field of the Earth is the (fully normalized) spherical harmonic expansion. As such, the aim of gravity field modeling is to determine a set of spherical harmonic coefficients; they can be arranged as an unknown vector $\mathbf{m}$. Furthermore, additional unknown parameters can be included in the vector $\mathbf{m}$, e.g. data biases. The available satellite data can be represented as a vector $\mathbf{y}$. Then, a certain functional model has to be established that relates these two vectors to each other:

$\mathbf{y}=\Phi(\mathbf{m})$.

Depending on the actual definition of the vector $\mathbf{y}$, the relationship of Eq. 1 can be either linear or non-linear. For example, satellite accelerations can be related to the vector $\mathbf{m}$ linearly (Ditmar and van Eck van der Sluijs 2004), whereas for an observed satellite orbit this relation is, generally speaking, non-linear (see e.g. Reigber 1989). In any case, Eq. 1 allows a set of data to be simulated on the basis of a given set of spherical harmonic coefficients. It is important to notice that, in practice, the functional model of Eq. 1 may suggest rather advanced computations. For example, it has to account for not only the static gravity field but also for its temporal variations, even if the latter are not estimated.

In a non-linear functional model, the determination of the unknown vector $\mathbf{m}$ should start from a linearization. A certain realistic reference model $\mathbf{m}_{\mathbf{0}}$ should be specified and used to compute the corresponding model response $\mathbf{y}_{\mathbf{0}}$ :

$\mathbf{y}_{\mathbf{0}}=\Phi\left(\mathbf{m}_{\mathbf{0}}\right)$.

The required accuracy of such a computation depends on how accurate the data are. For example, in CHAMP data processing, short-term temporal gravity field variations caused by mass transport in the oceans and atmosphere are typically ignored; in GRACE data processing, taking them into account is essential.

As long as the reference model is sufficiently close to the true one, Eq. 1 can be approximated by the Taylor expansion where only the zero- and first-order terms are retained:

$\mathbf{y}=\mathbf{y}_{\mathbf{0}}+\mathbf{A}\left(\mathbf{m}-\mathbf{m}_{\mathbf{0}}\right)$,

with $\mathbf{A}$ the matrix of partial derivatives (or the design matrix):

$A_{i j}=\frac{\partial \Phi_{i}(\mathbf{m})}{\partial m_{j}} \mid \mathbf{m}=\mathbf{m}_{0}$.

The relationship of Eq. 3 yields a linear functional model

$\mathbf{d}=\mathbf{A x}$,

with $\mathbf{x}$ the model correction to be found: $\mathbf{x}=\mathbf{m}-\mathbf{m}_{\mathbf{0}}$, and d the residual observations: $\mathbf{d}=\mathbf{y}-\mathbf{y}_{\mathbf{0}}$. Correspondingly, the final model can be computed at the last stage of data processing as $\mathbf{m}=\mathbf{m}_{\mathbf{0}}+\mathbf{x}$.

In fact, it is advisable to operate with residual observations even if the functional model is linear. This is because the accuracy and resolution of any satellite data set are limited. For example, it will be always impossible to recover 
high-frequency components of the gravity field (say, above degree 300 or slightly larger). Splitting observations into the reference model response and the residual part allows one to circumvent this problem. In this way, the final model is assembled of two ingredients: $\mathbf{m}_{\mathbf{0}}$, which may contain all the knowledge about the gravity field collected prior to a satellite mission, and $\mathbf{x}$, which contains only such corrections that are needed to make the final gravity field model fit to the newly acquired satellite data.

Assuming that noise in observations is Gaussian, an estimation of the unknown vector $\mathbf{x}$ can be obtained from the linear functional model of Eq. 5 with the BLUE (best linear unbiased estimator) concept (see, e.g.Teunissen 2000):

$\hat{\mathbf{x}}=\mathbf{N}^{-1} \mathbf{A}^{\mathrm{T}} \mathbf{C}_{\mathbf{d}}^{-1} \mathbf{d}$,

where $\mathbf{N}$ is the normal matrix:

$\mathbf{N}=\mathbf{A}^{\mathrm{T}} \mathbf{C}_{\mathbf{d}}^{-1} \mathbf{A}+\mathbf{C}_{\mathbf{m}_{\mathrm{o}}}^{-1}$,

$\mathbf{C}_{\mathbf{d}}$ is the data covariance matrix, and $\mathbf{C}_{\mathbf{m}_{\mathbf{0}}}$ is the covariance matrix of the reference model.

If the covariance matrix of the reference model is not available, a Tikhonov regularization matrix can be used instead (Tikhonov and Arsenin 1977). The regularization matrix must be supplied with an additional scaling factor - a regularization parameter. A discussion on the optimal choice of the regularization parameter in the context of global gravity field modeling can be found in Koch and Kusche 2002; Kusche and Klees 2002 and Ditmar et al. 2003. In this paper, however, we will focus on the covariance matrix of data noise $\mathbf{C}_{\mathrm{d}}$.

\section{Noise model}

\subsection{Definition}

To begin with, assume that the data form an uninterrupted time series (e.g. a complete series of satellite accelerations projected onto the radial direction). Later, we will make necessary remarks regarding data with gaps. We will not consider specially multi-component data sets. A generalization of the proposed methodology to such data is straightforward (at least, if different components can be considered uncorrelated with each other).

Data noise is usually time correlated. Even if correlations are absent in raw measurements, they may be introduced in the course of data pre-processing (e.g. noise in "observed" satellite accelerations is correlated even if noise in the orbit is not). A frequent assumption about noise is that it is stationary, i.e., loosely speaking, that its stochastic properties do not change with time. Then, the data covariance matrix is Toeplitz.

Such an assumption makes the stochastic description easier, but is not realistic in many cases. For example, orbits of satellites dedicated to gravity field studies are derived from data acquired by the on-board GPS receiver. Since the constellation of visible GPS satellites changes quickly for a lowEarth orbiter (LEO) in relation to ground-based GPS, the
LEO orbit accuracy may vary dramatically in time. Furthermore, a kinematic orbit may contain jumps or spikes (e.g., due to a change in the number of visible satellites). Obviously, satellite accelerations or other quantities derived from such an orbit will contain highly non-stationary noise.

To combine non-stationarity of noise with possible correlations in time, we propose to define the uninterrupted data covariance matrix $\mathbf{C}_{\mathbf{d}}^{\text {(ui) }}$ as follows:

$\mathbf{C}_{\mathbf{d}}^{(\text {ui) } \stackrel{\text { def }}{=} \mathbf{P C}} \mathbf{C}^{(\mathrm{nst})} \mathbf{P}^{\mathrm{T}}$,

where $\mathbf{C}^{(\text {nst })}$ is a diagonal matrix of size $N_{\text {ui }}^{\prime} \times N_{\text {ui }}^{\prime} ; \mathbf{P}$ is a rectangular matrix of size $N_{\mathrm{ui}} \times N_{\mathrm{ui}}^{\prime}$ obtained from a band-limited Toeplitz matrix by a corresponding "horizontal extension" (see Fig. 1); $N_{\mathrm{ui}}$ is the number of data, and $N_{\mathrm{ui}}^{\prime}$ is the number of data plus the number of non-zero diagonals in $\mathbf{P}$ minus 1 .

The matrix $\mathbf{C}^{(\mathrm{nst})}$ is responsible for the non-stationarity of the noise model, whereas the matrix $\mathbf{P}$ introduces correlations. The definition of Eq. 8 resembles the noise propagation formula and, therefore, can be interpreted as the assumption that actual noise is produced from non-stationary, non-correlated noise (with the covariance matrix $\mathbf{C}^{(\mathrm{nst})}$ ) by means of the linear transformation given by the matrix $\mathbf{P}$. In the absence of non-stationary noise (i.e. when the matrix $\mathbf{C}^{(\mathrm{nst})}$ is unit), the covariance matrix $\mathbf{C}_{\mathbf{d}}^{(\mathrm{ui})}$ becomes Toeplitz.

We would like to stress, however, that the matrix $\mathbf{P}$ is introduced on a purely formal basis; in general, one should not try to find a physical meaning of this matrix. In the next section, we will discuss how entries of the matrix $\mathbf{P}$ can be estimated.

As far as the non-stationary part $\mathbf{C}^{(\mathrm{nst})}$ is concerned, we will assume that it is given a priori. We believe that this assumption is reasonable, because in most cases the measurements are supplied with error estimates, which give an idea of how the measurement accuracy changes with time. The only elements of the matrix $\mathbf{C}^{(\mathrm{nst})}$ that are not available in this way are the elements at the edges, which have to be included as the result of the extension of the matrix $\mathbf{P}$ and, consequently, of the matrix $\mathbf{C}^{(\mathrm{nst})}$. We propose to set these elements equal to $\sigma^{2}$, the average value of the available diagonal elements of the matrix $\mathbf{C}^{\text {(nst) }}$. Furthermore, the matrix $\mathbf{P}$ still has to be defined. Therefore, we can assume - without loss of generality - that $\sigma^{2}=1$ : the necessary scaling can be assigned to the matrix $\mathbf{P}$. This means that the unavailable diagonal elements of the matrix $\mathbf{C}^{(\text {nst) }}$ can simply be set equal to unity.

Now consider the case of data with gaps. A data set $\mathbf{d}$ with gaps can be obtained from an uninterrupted data set $\mathbf{d}^{\text {(ui) }}$ by applying the "mask matrix" $\mathbf{M}$ :

$\mathbf{d}=\mathbf{M d}^{(\mathrm{ui})}$.

The mask matrix is the matrix obtained from the unit matrix by removing the rows that correspond to the missing data. Then, the data covariance matrix $\mathbf{C}_{\mathbf{d}}$ can be obtained from the uninterrupted data covariance matrix by means of the law of noise propagation:

$\mathbf{C}_{\mathbf{d}}=\mathbf{M C}_{\mathbf{d}}^{(\mathrm{ui})} \mathbf{M}^{\mathrm{T}}$ 

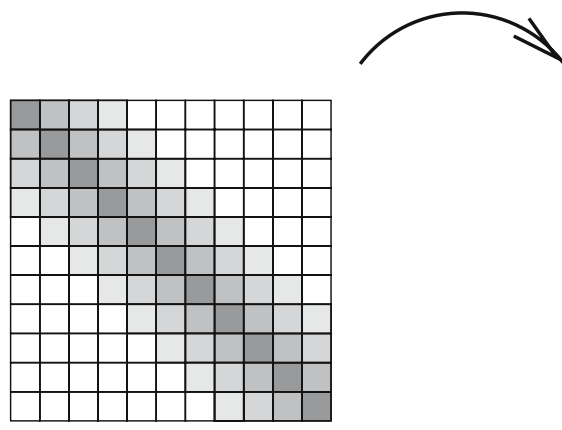

Fig. 1 Horizontal extension of a Toeplitz matrix

Notice that a gap in the data series is, most probably, accompanied by a gap in the time series of primary measurement errors. This means that more elements of the matrix $\mathbf{C}^{\text {(nst) }}$ are not available a priori than in the case of uninterrupted data set. These elements can be filled in the same way as the unavailable elements at the edges.

\subsection{Estimation}

To estimate the noise model, a noise realization is needed. In practice, however, a noise realization is typically not available. Therefore, we propose instead to use a posteriori residuals (the differences between the original and adjusted observations). This means that the gravity field has to be computed at least twice: the first model, produced without careful data weighting, is preliminary, and serves only for an estimation of a posteriori residuals; the second gravity field model that exploits the proper noise model is the final product.

In general, such an approach may be somewhat dangerous because the estimation obtained this way is biased. In the extreme case, where the number of unknown parameters equals the number of observations, the a posteriori residuals may even vanish, independent of the actual noise level. Our experience tells us, however, that in global gravity field modeling, this approach leads to very reasonable results (Liu et al. 2006). This can be explained by the fact that the number of observations typically exceeds the number of unknown parameters by the orders of magnitude.

The practical objective of the estimation procedure is to define the matrix $\mathbf{P}$ (cf. Eq. 8), which is the only ingredient of the noise model that is not given a priori. To reach this objective, two auxiliary steps are needed: (i) the estimation of the noise auto-covariance, and (ii) the estimation of the noise PSD.

\subsubsection{Noise auto-covariance}

First of all, let us neglect the non-stationarity of the noise. To make this assumption more realistic, the epochs when errors of primary measurements are large can be excluded from the

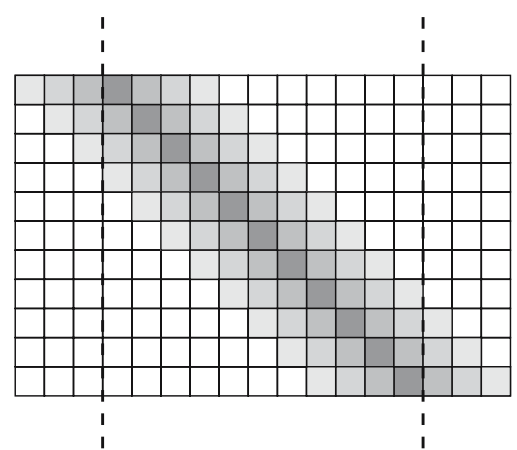

estimation procedure. Then, the data covariance matrix can be approximated as

$\mathbf{C}_{\mathbf{d}} \approx \mathbf{M C}^{(\mathbf{s t})} \mathbf{M}^{\mathrm{T}}$,

where $\mathbf{C}^{(\mathbf{s t})}$ is a covariance matrix of stationary noise, i.e., a Toeplitz matrix.

Let us denote the covariance between epochs $j$ and $j \pm k$ as $c_{k}$, an element of the auto-covariance vector with index $k$. By definition,

$c_{k}=E\left[n_{j} n_{j \pm k}\right]$,

where $E[\cdot]$ is the expectation operator; $n_{j}$ and $n_{j \pm k}$ is noise at two epochs separated by lag $k$.

Assuming that noise under consideration is a realization of an ergodic process (i.e. that averaging over noise realizations can be replaced by averaging in the time domain), we can turn Eq. 12 into a practical formula for the estimation of the auto-covariance vector:

$c_{k}=\frac{1}{N_{k}} \sum_{j} n_{j} n_{j \pm k} \quad\left(0 \leq k \leq N_{\mathrm{a}}\right)$,

where $N_{\mathrm{a}}$ is the maximum lag for which the auto-covariance is estimated, and $N_{k}$ is the number of pairs of elements that are used in the estimation of the $k$-th auto-covariance element. Notice that both elements of each pair should have indices in the interval $(1, N)$, where $N$ is the total length of a noise realization. Furthermore, some pairs with proper indices may be discarded if a noise realization is replaced by a posteriori residuals because such a series may contain interruptions related to gaps in the original data. In practice, it is advisable to choose $N_{\mathrm{a}}<N / 10$ (Klees and Broersen 2002).

As soon as the auto-covariance vector is estimated, (an estimation of) the covariance matrix $\mathbf{C}^{(\mathrm{st})}$ can be fully assembled: 


$$
\mathbf{C}^{(\mathrm{st})}=\left(\begin{array}{ccccccccccc}
c_{0} & c_{1} & \cdots & c_{N_{\mathrm{a}}} & & & & & & \\
c_{1} & c_{0} & c_{1} & \cdots & c_{N_{\mathrm{a}}} & & & & 0 & \\
\vdots & \ddots & \ddots & \ddots & & \ddots & & & & \\
c_{N_{\mathrm{a}}} & \cdots & c_{1} & c_{0} & c_{1} & \cdots & c_{N_{\mathrm{a}}} & & & \\
& \ddots & & \ddots & \ddots & \ddots & & \ddots & & \\
& & \ddots & & \ddots & \ddots & \ddots & & \ddots & \\
& & & c_{N_{\mathrm{a}}} & \cdots & c_{1} & c_{0} & c_{1} & \cdots & c_{N_{\mathrm{a}}} \\
& & & & \ddots & & \ddots & \ddots & \ddots & \vdots \\
& & & & & c_{N_{\mathrm{a}}} & \cdots & c_{1} & c_{0} & c_{1} \\
& & & & & & c_{N_{\mathrm{a}}} & \cdots & c_{1} & c_{0}
\end{array}\right)
$$

\subsubsection{Power spectral density}

The next stage is to estimate the noise PSD $u(f)$, where $f$ is the frequency. We would like to remark that this step is essential even if noise is stationary: it allows one to check that the covariance matrix is positive definite. The presence of the non-stationary part $\mathbf{C}^{(\mathbf{n s t})}$ is another reason to compute the PSD. Finally, an efficient application of the inverse covariance matrix to a vector is the third reason to do so.

In the discrete representation, elements of the PSD vector are defined as

$u_{m}=\lim _{N \rightarrow \infty} \frac{1}{\Delta f} E\left[s_{m} \bar{s}_{m}\right] \quad(0 \leq m<N)$,

where $s_{m}$ are the elements of a noise series spectrum: $\mathbf{s}=$ $1 / N \mathbf{F}^{*} \mathbf{n}$, with $\mathbf{F}$ being the matrix of the discrete Fourier transform (see the Appendix) and $\mathbf{F}^{*}$ the Hermitian conjugate (transposed complex conjugate) of $\mathbf{F} ; \bar{s}_{m}$ is the complex conjugate of $s_{m}$; and $\Delta f$ is the sampling interval in the frequency domain: $\Delta f=1 /(N \Delta t)$ with $\Delta t$ the sampling interval in the time domain.

In practice, the direct utilization of Eq. 15 for the estimation of the noise PSD may be problematic due to gaps in the available noise series. Therefore, we advise to estimate the PSD in an alternative way - on the basis of the Fourier transform of the auto-covariance (Kay and Marple 1981):

$\mathbf{u} \approx \frac{1}{\Delta f_{\mathrm{a}}} \frac{1}{2 N_{\mathrm{a}}} \mathbf{F}^{*} \mathbf{c}$

where $\Delta f_{\mathrm{a}}$ is the sampling interval in the Fourier domain dictated by the length of the auto-covariance vector: $\Delta f_{\mathrm{a}}=$ $1 /\left(2 N_{\mathrm{a}} \Delta t\right)$.

It is important to notice that Eq. 16 requires the elements of the auto-covariance vector to form a periodic series (see the Appendix). To this end, the auto-covariance vector estimated according to Eq. 13 has to be supplied with $N_{\mathrm{a}}-1$

additional elements that mirror the original ones: $c_{N_{\mathrm{a}}+k} \stackrel{\text { def }}{=}$ $c_{N_{\mathrm{a}}-k}\left(k=1,2, \ldots, N_{\mathrm{a}}-1\right)$. Furthermore, Eq. 16 is sufficiently accurate only when the correlation length is much less than $N_{\mathrm{a}}$. Unfortunately, this condition can hardly be met in practice. This is not only due to a slow decay of correlations in time, but also due to the presence of systematic distortions in the data (e.g. due to tidal effects that are not removed entirely because of a limited accuracy of tide models). Then, the PSD estimated with Eq. 16 may even become negative at some frequencies. This, of course, contradicts the definition of PSD (cf. Eq. 15).

The solution we propose is to truncate the original autocovariance vector by multiplying it with a function that rapidly decreases with time:

$\tilde{c}_{k} \stackrel{\text { def }}{=} c_{k} w_{k} \quad\left(0 \leq k \leq N_{\mathrm{a}}\right)$.

Such a truncation is equivalent in the Fourier domain to the cyclic convolution of the PSD with the Fourier transform of the vector w. As a result, the PSD becomes smoother. Thus, the situation is fully analogous to low-pass filtering of a signal, which can be achieved by a truncation of the signal spectrum (see the Appendix). In practice, we define the truncation function $w_{k}$ as a Gaussian:

$w_{k}=\mathrm{e}^{-\frac{(k \Delta t)^{2}}{2 Q^{2}}}$

Of course, other definitions can also be used.

The maximum possible half-width $Q$ of the truncation function is determined by trial and error. The procedure starts from a very large $Q$ and gradually decreases it until the PSD becomes positive at all the frequencies. Obviously, a sufficiently small $Q$ will always lead to a positive PSD. Indeed, a small $Q$ will make all the elements of the auto-covariance vector small except for the element with index zero: it will remain unchanged (in other words, the PSD will resemble the $\delta$-function). Since the zero element is positive by definition, application of the Fourier transform to such an auto-covariance vector will result in a positive, nearly constant spectrum.

\subsubsection{Matrix $\mathbf{P}$}

Equation 16 holds for an arbitrary long auto-covariance vector. The only effect of an increasing vector length is shortening the PSD sampling rate in the Fourier domain. Suppose that the PSD is re-sampled such that the new length $N_{\text {ui }}^{\prime}$ of the estimated vector $\mathbf{u}$ exceeds the number of data $N$ : $N_{\mathrm{ui}}^{\prime}=N_{\mathrm{ui}}+2 N_{\mathrm{e}}$, where $N_{\mathrm{e}}$ is an arbitrary number equal to or exceeding the maximum index of a non-zero element in the auto-covariance vector. Next, let us build up a diagonal matrix $\mathbf{U}$ composed of the elements of vector $\mathbf{u}$. Then, let us consider a new matrix $\mathbf{C}^{(\text {(circ) }}$ of size $N_{\text {ui }}^{\prime} \times N_{\text {ui }}^{\prime}$ defined as

$\mathbf{C}^{(\text {circ })} \stackrel{\text { def }}{=} \Delta f^{\prime} \mathbf{F} \mathbf{U} \mathbf{F}^{*}$,

where $\Delta f^{\prime}=1 /\left(N_{\mathrm{ui}}^{\prime} \Delta t\right)$.

Matrix $\mathbf{C}^{(\text {circ })}$ is circulant, and its first row is the result of the inverse Fourier transform applied to the vector $\Delta f^{\prime} \mathbf{u}$ (see the Appendix). Since the discrete Fourier transform is reversible, the first row of the matrix $\mathbf{C}^{(\mathrm{circ})}$ is nothing but the auto-covariance vector (extended with a sufficient amount of zeroes and with the "mirror elements", and cyclically reordered such that it starts from the zeroth element). Thus, the whole matrix $\mathbf{C}^{\text {(circ) }}$ can be understood as a cyclic extension of the estimated covariance matrix $\mathbf{C}^{\text {(st) }}$.

Let us introduce a "truncation matrix" $\mathbf{M}^{(\mathbf{t r})}$ of size $N_{\mathrm{ui}} \times$ $N_{\mathrm{ui}}^{\prime}$, obtained from the unit matrix by removing the first and 

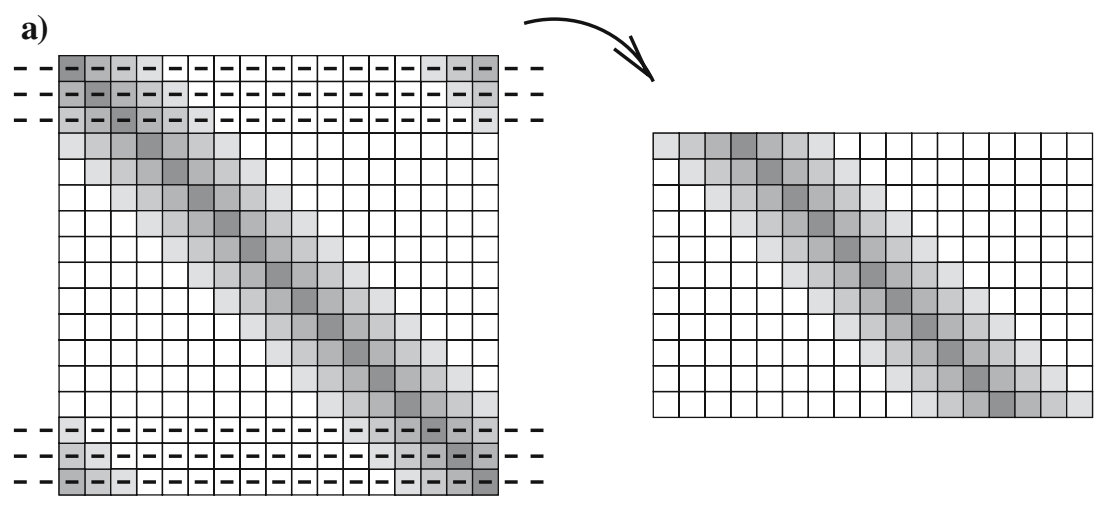

b)
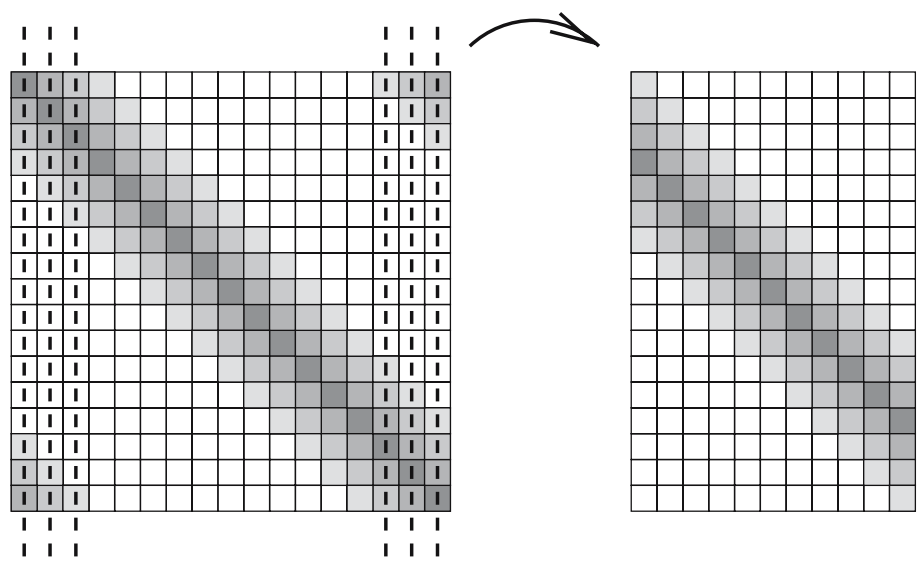

c)
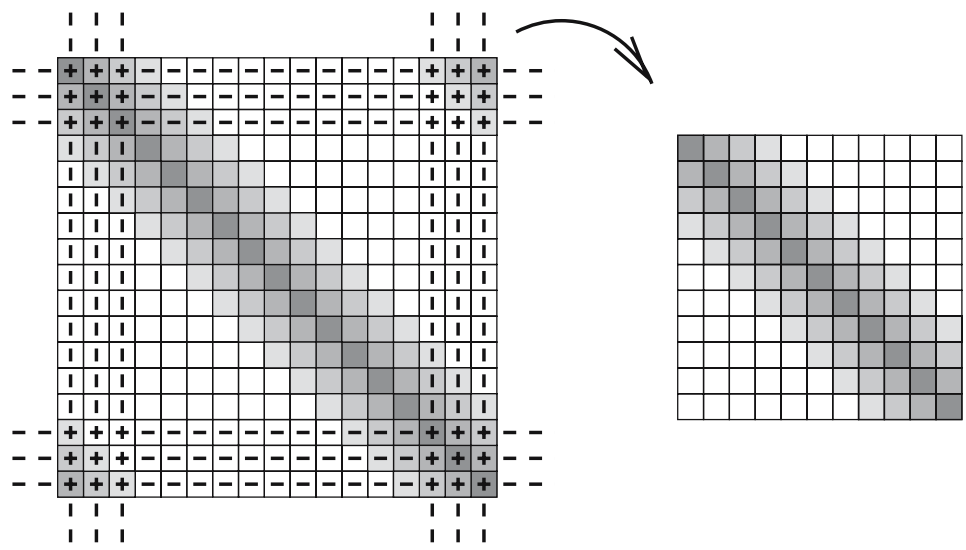

Fig. 2 Truncation of the circulant matrix $\mathbf{C}$ with the truncation matrix $\mathbf{M}^{(\mathrm{tr})}$. $\mathbf{a} \mathbf{M}^{(\mathrm{tr})} \mathbf{C}$ is a band-limited Toeplitz matrix extended horizontally; b $\mathbf{C}\left(\mathbf{M}^{(\mathrm{tr})}\right)^{\mathrm{T}}$ is a band-limited Toeplitz matrix extended vertically; $\mathbf{c} \mathbf{M}^{(\mathrm{tr})} \mathbf{C}\left(\mathbf{M}^{(\mathrm{tr})}\right)^{\mathrm{T}}$ is a square band-limited Toeplitz matrix

last $N_{\mathrm{e}}$ rows. Then, the matrix $\mathbf{C}^{(\mathrm{st})}$ can be formally related to the matrix $\mathbf{C}^{\text {(circ) }}$ as follows (see Fig. 2):

$\mathbf{C}^{(\mathrm{st})}=\mathbf{M}^{(\mathrm{tr})} \mathbf{C}^{(\mathrm{circ})}\left(\mathbf{M}^{(\mathrm{tr})}\right)^{\mathrm{T}}$.

Notice that Eq. 20 can easily be generalized to the case of data with gaps: it is enough to remove in the matrix $\mathbf{M}^{\text {(tr) }}$ also the rows related to the missing observations.

Since the proposed procedure guarantees that all elements of the PSD vector are positive, the square root of the matrix $\mathbf{C}^{(\text {(irc) }}$ can be introduced (see the Appendix):

$$
\left(\mathbf{C}^{(\text {circ })}\right)^{\frac{1}{2}}=\sqrt{\frac{\Delta f^{\prime}}{N_{\mathrm{ui}}^{\prime}}} \mathbf{F U}^{\frac{1}{2}} \mathbf{F}^{*} .
$$

Then, Eq. 20 can be re-written as

$\mathbf{C}^{(\mathbf{s t})}=\mathbf{Q Q}^{\mathrm{T}}$,

where

$\mathbf{Q} \stackrel{\text { def }}{=} \mathbf{M}^{(\mathrm{tr})}\left(\mathbf{C}^{(\mathrm{circ})}\right)^{\frac{1}{2}}$.

The matrix $\mathbf{Q}$ is a band-limited Toeplitz matrix extended horizontally (Fig. 2a). Comparison of Eqs. 8 and 22 leads 
to the conclusion that these formulae coincide for stationary noise (i.e. when the matrix $\mathbf{C}^{(\mathbf{n s t})}$ is a unit matrix) provided that

$\mathbf{P} \stackrel{\text { def }}{=} \mathbf{Q}$.

It is, therefore, natural to define the matrix $\mathbf{P}$ this way in all cases, whether the noise is stationary or not. Thus, we have

$\mathbf{C}_{\mathbf{d}}=\mathbf{Q C}^{(\mathrm{nst})} \mathbf{Q}^{\mathrm{T}}$.

It is easy to see that the data covariance matrix obtained in this way is always positive definite, including the case of data with gaps. Consider the expression

$\mathbf{y}^{\mathrm{T}} \mathbf{C}_{\mathbf{d}} \mathbf{y}=\mathbf{y}^{\mathrm{T}} \mathbf{Q} \mathbf{C}^{(\mathrm{nst})} \mathbf{Q}^{\mathrm{T}} \mathbf{y}=\mathbf{z}^{\mathrm{T}} \mathbf{C}^{(\mathrm{nst})} \mathbf{z}$,

where $\mathbf{y}$ is an arbitrary vector, and

$\mathbf{z}=\mathbf{Q}^{\mathrm{T}} \mathbf{y}$.

Let us show that if the vector $\mathbf{y}$ is non-zero, the vector $\mathbf{z}$ is also non-zero. From Eqs. 21, 23 and 27, it follows that

$\mathbf{z}=\sqrt{\frac{\Delta f^{\prime}}{N_{\mathrm{ui}}^{\prime}}} \mathbf{F} \mathbf{U}^{\frac{1}{2}} \mathbf{F}^{*}\left(\mathbf{M}^{(\mathbf{t r})}\right)^{\mathrm{T}} \mathbf{y}$.

We have to demonstrate that the application of any matrix from Eq. $28-\mathbf{F}, \mathbf{U}^{\frac{1}{2}}, \mathbf{F}^{*}$, and $\left(\mathbf{M}^{(\mathbf{t r})}\right)^{\mathrm{T}}-$ to a non-zero vector results in a non-zero vector (i.e. that all these matrices have the full column rank).

For matrices $\mathbf{F}$ and $\mathbf{F}^{*}$ this is obvious; otherwise, the discrete Fourier transform would not be a reversible operation. This is also obvious for the matrix $\mathbf{U}^{\frac{1}{2}}$ because this is a diagonal matrix with all the diagonal elements greater than zero (we make use of the fact that the estimated PSD is forced to be positive at all the frequencies). Finally, it is easy to see that the matrix $\left(\mathbf{M}^{(\mathrm{tr})}\right)^{\mathrm{T}}$ just stretches a vector of size $N_{\mathrm{ui}}$ to the size $N_{\text {ui }}^{\prime}$ by adding some zero elements. All the elements from the original vector are preserved, so that the output vector is non-zero, if the input vector is non-zero.

Next, the matrix $\mathbf{C}^{(\mathrm{nst})}$ is positive definite by definition. Therefore, for any non-zero vector $\mathbf{y}$ we have the relationship

$\mathbf{y}^{\mathrm{T}} \mathbf{C}_{\mathbf{d}} \mathbf{y}=\mathbf{z}^{\mathrm{T}} \mathbf{C}^{(\text {(nst) }} \mathbf{z}>0$,

which completes the proof.

\subsection{Usage of a noise model in data processing}

As follows from Eqs. 6 and 7, the data processing deals with the inverse data covariance matrix. Since this matrix is usually huge, its explicit inversion is impossible. Therefore, it is necessary to discuss how an accurately estimated data covariance matrix can be used in practical data processing. The algorithm we propose is a further development of the ones described in (Klees and Ditmar 2004; Ditmar and van Eck van der Sluijs 2004; Ditmar et al. 2006b).

First of all, it is advisable to solve the system of normal equations (Eq. 6) with the pre-conditioned conjugate gradient (PCCG) method (Hestenes and Stiefel 1952) as it was proposed in the context of global gravity field modeling by Schuh (1996). As such, it is not necessary to compute the normal matrix explicitly. Instead, it is sufficient to have a procedure for the multiplication of the normal matrix to a vector. In such a procedure, all the matrices that compose the normal matrix (cf. Eq. 7) have to be multiplied with a vector one-by-one. This concerns, in particular, the matrix $\mathbf{C}_{\mathbf{d}}^{-1}$.

Thus, it is not necessary to invert the data covariance matrix; it is enough to solve the system of linear equations based on this matrix a number of times. For this purpose, a "lowlevel" PCCG procedure can be used, which has to be executed at each iteration of the "high-level" PCCG procedure. To make the low-level PCCG procedure accurate and numerically efficient, one has to implement two basic operations: (i) the exact multiplication of the data covariance matrix to a vector, and (ii) a pre-conditioning, i.e., an approximate multiplication of the inverse data covariance matrix to a vector.

\subsubsection{Multiplication of the data covariance matrix to a vector}

According to Eq. 25, the multiplication of the matrix $\mathbf{C}_{\mathbf{d}}$ to a given vector $\mathbf{z}$ can be performed as a sequential multiplication of matrices $\mathbf{Q}^{\mathrm{T}}, \mathbf{C}^{(\mathrm{nst})}$, and $\mathbf{Q}$ to vectors.

In principle, each of the three multiplications can be done explicitly. The problem is, however, that the number of nonzero elements in each row/column of the matrix $\mathbf{Q}$ can be large (it depends on how much the auto-covariance vector is truncated; cf. Eq. 17). Since the matrix $\mathbf{Q}$ has to be multiplied with a vector many times, this step may slow down the whole data processing procedure significantly.

The solution we propose is to make use of the representation of Eq. 23. That is, this step can be implemented as a multiplication of two matrices to a vector: $\left(\mathbf{C}^{(\text {circ })}\right)^{\frac{1}{2}}$ and $\mathbf{M}^{(\mathrm{tr})}$. The first matrix is circulant; its application to a vector is nothing but filtering (or, more strictly, cyclic convolution). This operation can be efficiently implemented in the Fourier domain (see Eq. 21 and the Appendix).

As far as the matrix $\mathbf{M}^{(\mathrm{tr})}$ is concerned, its application to a vector reduces to eliminating edge elements as well as elements that correspond to data gaps, if they exist. The application of the matrix $\mathbf{Q}^{\mathrm{T}}$ to a vector can be implemented in a similar way.

\subsubsection{Pre-conditioning}

According to Eqs. 23 and 25, the data covariance matrix can be represented as

$\mathbf{C}_{\mathbf{d}}=\mathbf{M}^{(\mathrm{tr})} \mathbf{B}\left(\mathbf{M}^{(\mathrm{tr})}\right)^{\mathrm{T}}$

where $\mathbf{B}$ is a matrix of size $N_{\mathrm{ui}}^{\prime} \times N_{\mathrm{ui}}^{\prime}$ :

$\mathbf{B}=\left(\mathbf{C}^{(\mathrm{circ})}\right)^{\frac{1}{2}} \mathbf{C}^{(\mathrm{nst})}\left(\mathbf{C}^{(\mathrm{circ})}\right)^{\frac{1}{2}}$.

The inverse of a circulant matrix can be found with ease (see the Appendix). Therefore, obtaining the inverse of $\mathbf{B}$ is also straightforward: 
$\mathbf{B}^{-1}=\left(\mathbf{C}^{(\mathrm{circ})}\right)^{-\frac{1}{2}}\left(\mathbf{C}^{(\mathrm{nst})}\right)^{-1}\left(\mathbf{C}^{(\mathrm{circ})}\right)^{-\frac{1}{2}}$,

where

$$
\left(\mathbf{C}^{(\mathrm{circ})}\right)^{-\frac{1}{2}}=\sqrt{\frac{N_{\mathrm{ui}}^{\prime}}{\Delta f^{\prime}}} \frac{1}{\left(N_{\mathrm{ui}}^{\prime}\right)^{2}} \mathbf{F} \mathbf{U}^{-\frac{1}{2}} \mathbf{F}^{*} .
$$

Then, the pre-conditioner $\mathbf{G}$ - an approximate inverse of $\mathbf{C}_{\mathbf{d}}$ - can be defined as

$\mathbf{G} \stackrel{\text { def }}{=} \tilde{\mathbf{M}}^{(\mathrm{tr})} \tilde{\mathbf{B}}^{-1}\left(\tilde{\mathbf{M}}^{(\mathrm{tr})}\right)^{\mathrm{T}}$.

Notice that both the truncation matrix and the matrix $\mathbf{B}$ are accompanied by a "tilde". This indicates, in this context, that the inversion of both matrices may have to be accompanied by an extra extension, as follows.

The number of non-zero diagonals in an inverse circulant matrix can be totally different from that in the original circulant matrix. On the other hand, we assume, as before, that the application of the truncation matrix totally removes nonzero elements (or, more strictly, not nearly-zero elements) in the upper-right and lower-left corners of a circulant matrix. Therefore, we have to take into account that the truncation matrix in the pre-conditioner $-\tilde{\mathbf{M}}^{(t r)}$ - may be different from the original one because it has to remove another (e.g. larger) number of rows/columns at the edges. Similarly, a different extension of the matrix $\mathbf{B}$ may be needed in the context of pre-conditioning.

Finally, we would like to remark that the composition of the pre-conditioner (Eq. 34), is similar to that of the data covariance matrix itself (Eq. 30). This means that the multiplication of the pre-conditioner to a vector can be done in exactly the same way as the multiplication of the data covariance matrix to a vector (namely, with filtering in the Fourier domain).

\section{Examples}

\subsection{CHAMP data processing}

We have used the above-proposed approach for modeling the Earth's gravity field from a 322-day set of CHAMP data provided by D. Švehla and M. Rothacher, Technical University of Munich (Švehla and Rothacher 2003). The data set includes a kinematic and a reduced-dynamic orbit of the CHAMP satellite. The functional model we exploit is based on the satellite accelerations that are derived from a kinematically determined satellite orbit and transformed into the local orbital reference frame (LORF) (Ditmar and van Eck van der Sluijs 2004).

The CHAMP data set also includes the covariance matrix of positioning errors; the matrix consists of $3 \times 3$ blocks, one block per epoch (no information about temporal correlations was considered). The provided information was used to build up the matrix $\mathbf{C}^{\text {(nst) }}$. Two different ways were tried: (i) the covariances (off-diagonal elements) were set equal to zero, so that the matrix $\mathbf{C}^{(\mathrm{nst})}$ is purely diagonal; and (ii) the covariances were preserved (in this case, an explicit inverse of each $3 \times 3$ block had to be computed for the purpose of pre-conditioning). Importantly, the provided covariance matrix was scaled so that the average variance per component (along-track, cross-track, and radial) became equal to one, as explained in Sect. 3.1.

For the purpose of gravity field modeling, residual satellite accelerations are derived from "observed" ones by subtracting the non-gravitational accelerations (measured with the on-board CHAMP accelerometer) and the reference accelerations. To compute the latter ones, we used the EGM96 static gravity field model (Lemoine et al. 1998) to degree and order 360, combined with models of astronomic, solid-Earth and oceanic tides (Ditmar et al. 2006b).

An outlier detection procedure was used in order to minimize the influence of jumps and spikes in the kinematic orbit (Ditmar et al. 2006b). Accelerometer scale factors and biases (one per component per 10-day interval) were explicitly estimated jointly with gravity field parameters. The overall data processing procedure consisted of the following steps: (i) preliminary gravity field modeling and computation of a posteriori residuals; (ii) estimation of the noise model; and (iii) final gravity field modeling.

The outcome of the modeling procedure is a set of fully normalized spherical harmonic coefficients from degree 2 to 70. The accuracy of final results was assessed by a comparison with the state-of-the-art gravity field model EIGENCG03C (Förste et al. 2005) truncated at degree 70. That model has been compiled from various satellite, airborne, and terrestrial data. In the range of degrees we are interested in, the dominant contributor to the EIGEN-CG03C model is a 376-day GRACE data set. Since these data are significantly more accurate than CHAMP data, discrepancies between a CHAMP-based model and EIGEN-CG03C can be safely interpreted as errors in a CHAMP-based model.

\subsubsection{Computation of a posteriori residuals}

In the preliminary gravity field modeling, we used an analytic definition of the noise square-root-PSD originally proposed by Ditmar and van Eck van der Sluijs (2004):

$$
\sqrt{u(f)}=\frac{\sigma}{(\Delta t)^{\frac{3}{2}}}\left[2(1-\cos (2 \pi f \Delta t))+\left(\frac{\Delta t}{\tau}\right)^{2}\right],
$$

where $f$ is the frequency, $\sigma$ is a scaling factor that can be interpreted as the average CHAMP positioning accuracy, and $\tau$ is a parameter that controls the behavior of the PSD at low frequencies.

Here, we set $\sigma$ equal to the a priori average CHAMP positioning accuracy for a given component, whereas $\tau$ was set equal to 180s. The latter value was selected on the basis of the numerical study of Ditmar and van Eck van der Sluijs (2004). In fact, this is not the optimal value. Ditmar et al. (2006b) showed later that a smaller $\tau$ (about 60s) can lead to better results. We made the choice of 180 s deliberately: to demonstrate that the proposed data processing scheme ultimately 
delivers reasonable results even if little a priori information exists about the noise model.

The specified noise square-root-PSD per component is shown in Fig. 3. The covariances between the components were taken into account. The existing covariance matrix of the EGM96 model was used at this stage as the covariance matrix $\mathbf{C}_{\mathbf{m}_{\mathbf{o}}}$ (cf. Eq. 7).

A comparison with the EIGEN-CG03C model showed that the RMS accuracy of the produced preliminary gravity model in terms of geoid heights is $19.5 \mathrm{~cm}$. This is noticeably worse than that for the DEOS_CHAMP-01C_70 model, $14.1 \mathrm{~cm}$ (Ditmar et al. 2006b). The difference can be explained by a more accurate noise model used to produce the DEOS_CHAMP-01C_70 model.

The residual gravity field model we have obtained (i.e. the total model minus the reference model) was used to compute a series of (residual) adjusted satellite accelerations. Furthermore, the adjusted accelerations were corrected for by taking into account estimated biases and accelerometer scale factors. Then, the series of a posteriori residuals obtained as the difference between the original and the adjusted residual accelerations was considered as a realization of data noise.

\subsubsection{Estimation of the noise model}

A noise PSD was computed on the basis of a posteriori residuals as explained in Sect. 3.2.2. The number of lags in the computation of the auto-covariance was set equal to $2^{15}+1$, which corresponds to the time interval of $32,769 \times 30 \mathrm{~s} \approx 273 \mathrm{~h}$ (a too short interval would lead to inaccuracies at low frequencies, whereas a too long interval would make the computations too time consuming).

To ensure the positiveness of the computed PSD, it was smoothed with $Q \approx 21 \mathrm{~h}$ for the along-track and cross-track components, and with $Q \approx 29 \mathrm{~h}$ for the radial component (Fig. 4). Furthermore, more drastically smoothed PSDs were obtained after setting $Q=3 \mathrm{~h}$ for all three components (Fig. 5). Finally, the square-root-PSDs were approximated by an analytic expression of Eq. 35 (Fig. 6).

As could be expected, a smaller factor $Q$ leads to a stronger truncation of the auto-covariance and, consequently, to

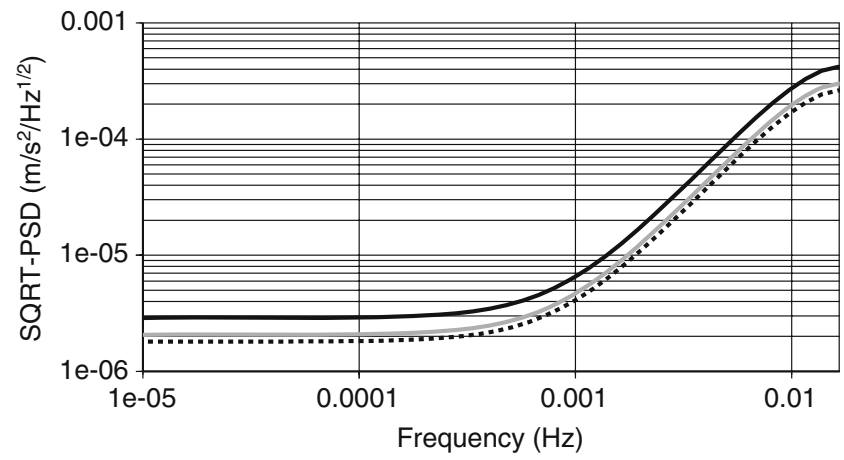

Fig. 3 Square-root noise PSD, as it was defined in the preliminary gravity field modeling. Solid black, gray, and dotted black lines correspond to the radial, along-track, and cross-track components, respectively

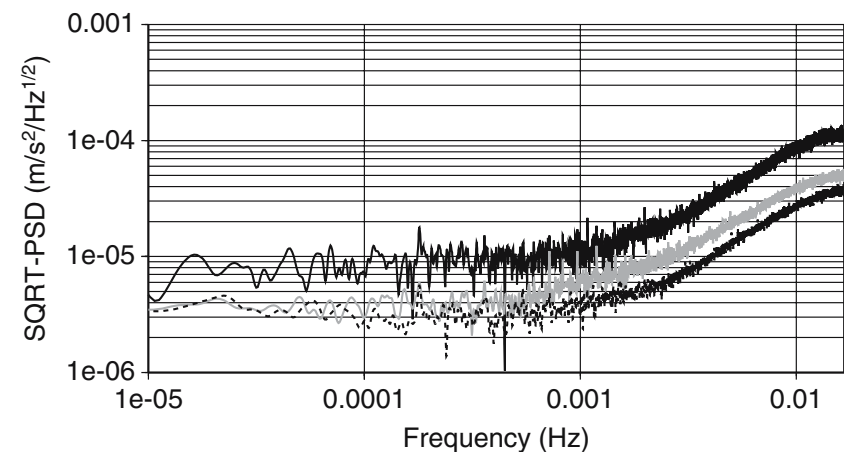

Fig. 4 Square-root PSD of noise in CHAMP accelerations. Solid black, gray, and dotted black lines correspond to the radial, along-track, and cross-track components, respectively. Smoothing applied is just enough to make the PSDs positive at all the frequencies $(Q \approx 29,21$, and $21 \mathrm{~h}$ for the three components, respectively)

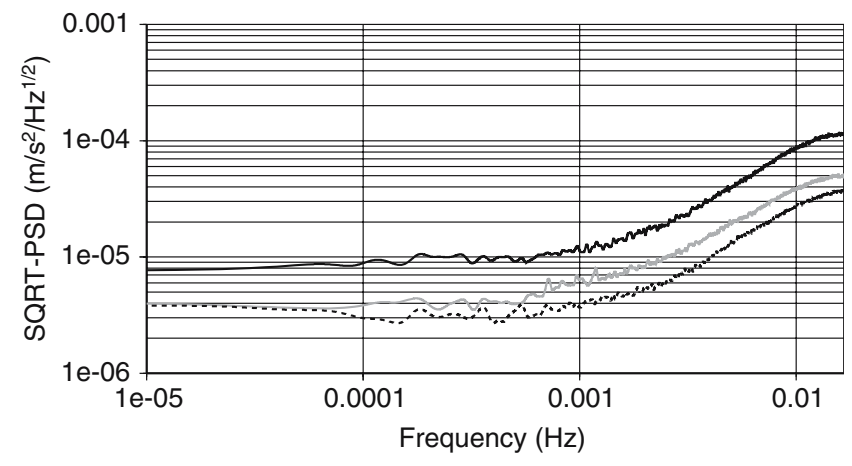

Fig. 5 Same as Fig. 4 but smoothing is stronger ( $Q=3 \mathrm{~h}$ for all the components)

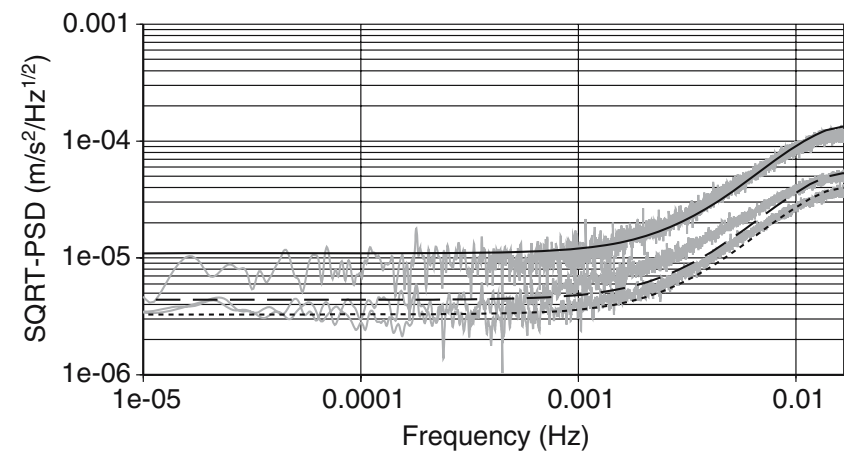

Fig. 6 Analytic approximation of the square-root PSD of noise in CHAMP accelerations. Solid, dashed, and dotted lines correspond to the radial, along-track, and cross-track components, respectively. Original square-root PSDs taken over from Fig. 4 are shown in gray as a reference. The chosen parameters of the analytic approximation are: $\sigma$ $=2,1.5$, and $5 \mathrm{~mm}$ for the three components, respectively; $\tau=50 \mathrm{~s}$ for all three components

a smoother PSD. In the case of the analytic approximation, the result is even smoother; only the major features of the noise behavior are captured: a nearly constant level at low frequencies and an increase at high frequencies. The three 
Table 1 Gravity field modeling from CHAMP accelerations

\begin{tabular}{|c|c|c|c|c|c|c|}
\hline \multirow[t]{2}{*}{ Noise model } & \multirow{2}{*}{$\begin{array}{l}\text { Co-variances } \\
\text { between the } \\
\text { components }\end{array}$} & \multirow[t]{2}{*}{ Regularization } & \multicolumn{4}{|c|}{ Cumulative geoid height error $(\mathrm{cm})$} \\
\hline & & & degree 10 & degree 20 & degree 40 & degree 70 \\
\hline Accurate numerical & No & No & 0.44 & 0.88 & 3.1 & 30.8 \\
\hline Smoothed numerical & No & No & 0.45 & 0.89 & 3.2 & 30.9 \\
\hline Analytic & No & No & 0.46 & 0.92 & 3.2 & 31.7 \\
\hline Accurate numerical & Yes & No & 0.45 & 0.89 & 3.1 & 30.9 \\
\hline Accurate numerical & No & Yes & 0.35 & 0.79 & 2.8 & 13.6 \\
\hline
\end{tabular}

Accuracy of resulting models in terms of cumulative geoid heights

sets of PSDs were used to build up three noise models. Hereafter, they are referred to as "accurate numerical", "smoothed numerical", and "analytic", respectively.

\subsubsection{Final gravity field modeling}

Each of the three noise models was exploited in gravity field modeling as discussed in Sect. 3. In the first instance, covariances between the components were ignored. Furthermore, the regularization matrix $\mathbf{C}_{\mathbf{m}_{0}}$ was set equal to zero. The quality of the produced models is compared in Table 1. One can see that the accurate numerical noise model leads to marginal improvements in comparison with the smoothed numerical one, whereas the latter is slightly better than the analytic noise model.

In the next step, we have included correlations between the components in the stochastic model. It turned out that the quality of the gravity field remained basically the same, or even became a bit lower (Table 1). In fact, worsening the model after taking the cross-component correlations is not very surprising because these correlations are not taken into account in the noise estimation procedure.

Finally, the regularization matrix, defined as the full covariance matrix of the EGM96 model (to degree and order 70), was added to the normal matrix. Naturally, this made the model quality significantly better (Table 1). It is worthwhile to note that the total RMS geoid height error (which is equal to the cumulative geoid height error from degree 2 to degree 70 ) is in this case better than that of the DEOS_CHAMP01C_70 model $(14.1 \mathrm{~cm})$.

This proves that a proper noise model may improve the results of gravity field modeling.

\subsection{CHAMP data processing in the absence of accelerometer data}

A common opinion is that high-precision measurements of non-gravitational accelerations are crucial for accurate gravity field modeling. On the contrary, Ditmar et al. (2006b) claimed that their gravity field modeling procedure is not sensitive to errors in accelerometer scale factors. Furthermore, Ditmar et al. (2004a) have shown that neglecting accelerometer data may lead to only minor additional errors in a derived gravity field model. Therefore, we found that it is important to analyze how much a gravity field model can be distorted in the absence of accelerometer data if a proper noise model is used.

To begin with, we have computed the PSD of non-gravitational accelerations measured by the accelerometer on-board the CHAMP satellite (Fig. 7). One can see that non-gravitational accelerations are relatively strong at very low frequencies (below $3 \times 10^{-5} \mathrm{~Hz}$ ). Furthermore, they show five prominent peaks in the range of frequencies between 0.0001 and $0.001 \mathrm{~Hz}$. These peaks correspond to the frequencies of $1,2, \ldots, 5$ cycles per revolution (cpr).

The peak at frequency $1 \mathrm{cpr}$ is especially visible at the along-track and the cross-track components. In the first case, it can be explained by variations of the atmospheric drag caused by the orbit eccentricity ( $e \approx 0.002$ ). The probable reason in the second case is the atmosphere rotation, so that the drag force is directed to the right on the ascending pass and to the left on the descending pass. The peak at frequency $2 \mathrm{cpr}$ is very strong only in the along-track component. It can be attributed to variations of the atmospheric drag caused by the Earth's flattening. Other peaks at frequencies less or equal to $5 \mathrm{cpr}$ seem to be comparable in magnitude with the noise level, whereas all the peaks at frequencies above $5 \mathrm{cpr}$ seem to be significantly below the noise level.

It is, therefore, tempting to conclude immediately that non-gravitational accelerations, even if they are not removed from the data, must not destroy a resulting gravity field model. Such a conclusion is, however, premature. The fact is that all the peaks at the plots have been somewhat smoothed. Without the smoothing, a peak magnitude can be significantly larger. This can be interpreted as an evidence that non-gravitational accelerations are not stochastic quantities. Therefore, it is necessary to perform gravity field modeling in order to draw conclusions about a possibly destructive role of non-gravitational accelerations.

The CHAMP accelerations not cleaned from non-gravitational signals were processed in exactly the same way as explained above, starting from the preliminary gravity field modeling. Naturally, the accelerometers' scale factors were excluded from the list of unknown parameters. The estimated noise PSD is shown in Fig. 8. One can see that non-gravitational accelerations significantly exceed the random noise 

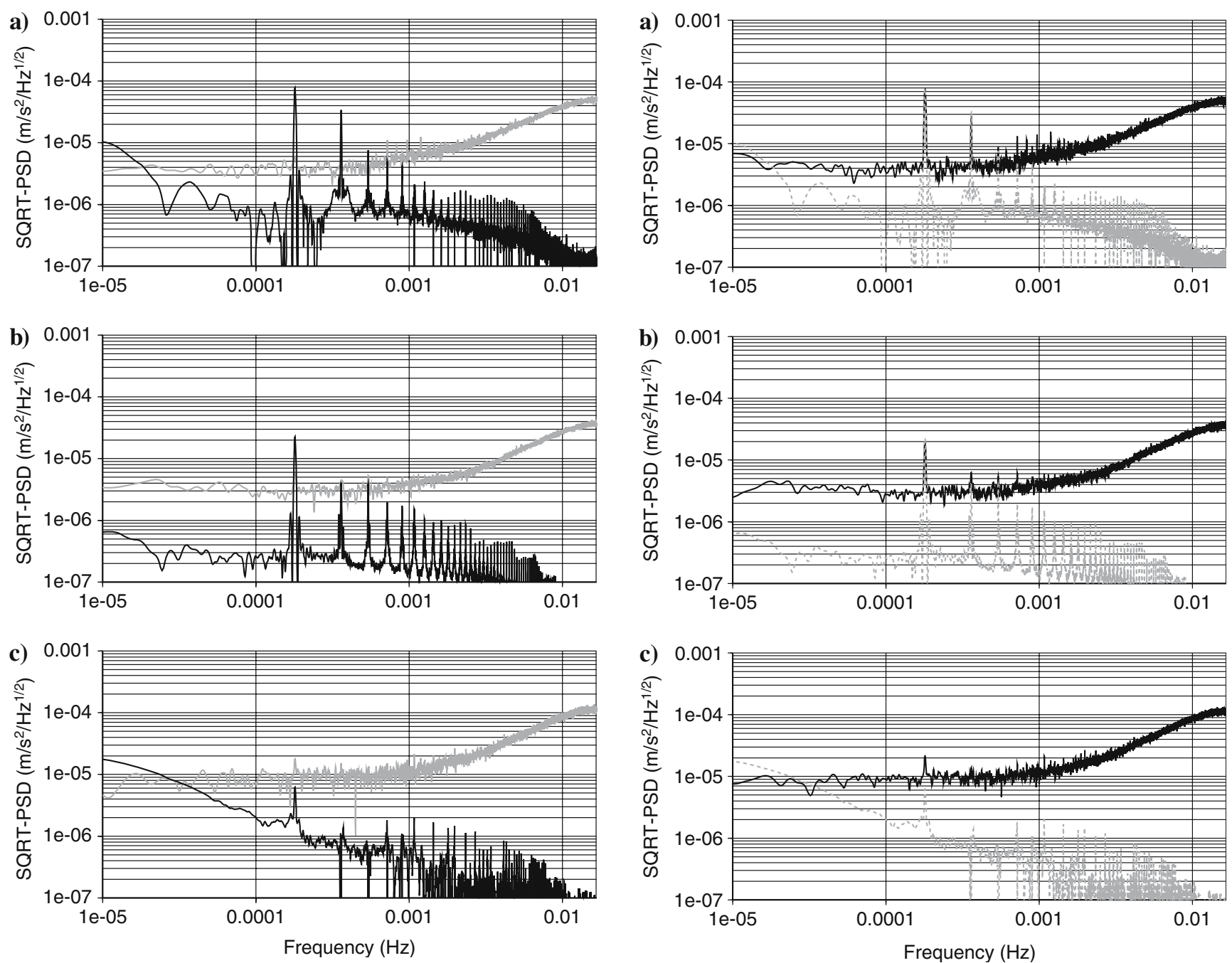

Fig. 7 Square-root PSD of non-gravitational CHAMP accelerations (in black). $\mathbf{a}$ the along-track component; $\mathbf{b}$ the cross-track component; $\mathbf{c}$ the radial component. In all the cases, smoothing with $Q=30 \mathrm{~h}$ is applied. The square-root PSD of noise in CHAMP accelerations is shown in gray as a reference

level at frequencies 1 and $2 \mathrm{cpr}$ at the along track-component and at the frequency $1 \mathrm{cpr}$ at the cross-track component. This results in prominent peaks in the a posteriori residuals. It is also interesting to notice that the along-track and radial components of the a posteriori residuals are weaker at low frequencies than the corresponding component of non-gravitational accelerations. This can be explained by the fact that the data biases, which are estimated over 10-day intervals, partly absorb the influence of non-gravitational accelerations.

As before, both the cross-component correlations and the regularization were, in the first instance, switched off. The estimated noise PSD obtained after a smoothing with $Q \approx$ $30 \mathrm{~h}$ in all three components (just enough to make the PSD positive at all the frequencies) was used as the basis for the accurate numerical noise model. Furthermore, an approximate numerical noise model was defined by smoothing with $Q=3 \mathrm{~h}$. Finally, the analytic noise model from the previ-

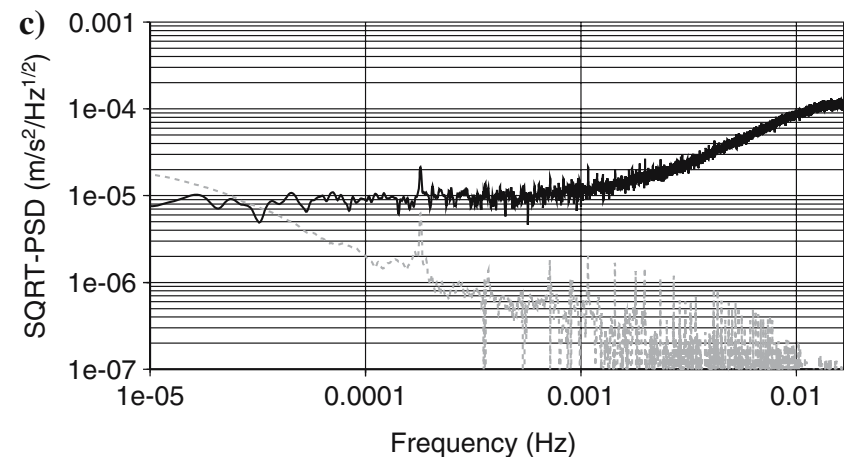

Fig. 8 Square-root PSD of noise in CHAMP accelerations not cleaned from non-gravitational accelerations (in black): a the along-track component; b the cross-track component; $\mathbf{c}$ the radial component. In all the cases, smoothing with $Q=30 \mathrm{~h}$ is applied. The square-root PSD of non-gravitational accelerations is shown as a dashed gray line as a reference

ous example was re-used (it was not necessary to update it because the noise PSD has not changed much apart from a limited number of peaks).

Each of these three noise models was used to produce a gravity field model. These gravity field models are compared in Table 2. As before, one can see that the accurate numerical noise model leads to the best results in terms of the total RMS geoid height error, whereas the analytic noise model performs slightly worse. It is interesting to notice that the smoothed numerical noise model gives the best results at low degrees; the reason for this is not entirely clear.

Furthermore, we have tried to take into account the correlations between the components. As before, the model quality does not change much in this case (Table 2). Finally, the gravity field model improves dramatically when the regularization is switched on. 
Table 2 Gravity field modeling from CHAMP accelerations not cleaned from non-gravitational accelerations

\begin{tabular}{|c|c|c|c|c|c|c|}
\hline \multirow[t]{2}{*}{ Noise model } & \multirow{2}{*}{$\begin{array}{l}\text { Co-variances } \\
\text { between the } \\
\text { components }\end{array}$} & \multirow[t]{2}{*}{ Regularization } & \multicolumn{4}{|c|}{ Cumulative geoid height error (cm) } \\
\hline & & & degree 10 & degree 20 & degree 40 & degree 70 \\
\hline Accurate numerical & No & No & 0.46 & 0.87 & 3.1 & 29.8 \\
\hline Smoothed numerical & No & No & 0.42 & 0.86 & 3.1 & 30.0 \\
\hline Analytic & No & No & 0.45 & 0.91 & 3.2 & 30.8 \\
\hline Accurate numerical & Yes & No & 0.49 & 0.89 & 3.0 & 29.7 \\
\hline Accurate numerical & No & Yes & 0.35 & 0.78 & 2.8 & 13.5 \\
\hline
\end{tabular}

Accuracy of resulting models in terms of cumulative geoid heights

It is interesting to compare models produced with and without accelerometer data (cf. Tables 1 and 2). It turns out that the absence of accelerometer data does not deteriorate the resulting gravity field model. Furthermore, the total RMS geoid height error even seems to reduce. The explanation for the latter effect is a slightly larger number of data that could be used in the absence of cleaning from non-gravitational accelerations. In the course of this cleaning, about $5 \%$ of data is lost due to the absence of either star camera measurements or the accelerometer measurements (Ditmar et al. 2006b).

Gravity field models obtained with and without accelerometer data can also be compared graphically (Fig. 9). Both models demonstrate practically the same accuracy at all degrees except for degree 2 , where the cleaned data definitely lead to a better result.

One can argue that non-gravitational accelerations must influence, first of all, the zonal spherical harmonic coefficients, for which a resonance effect may take place. Such an influence can hardly be noticed from Fig. 9, where the errors at all the orders for a given degree are summed up. Therefore, we have also compared the errors in zonal spherical harmonic coefficients alone (Fig. 10). Again, one can see that cleaning

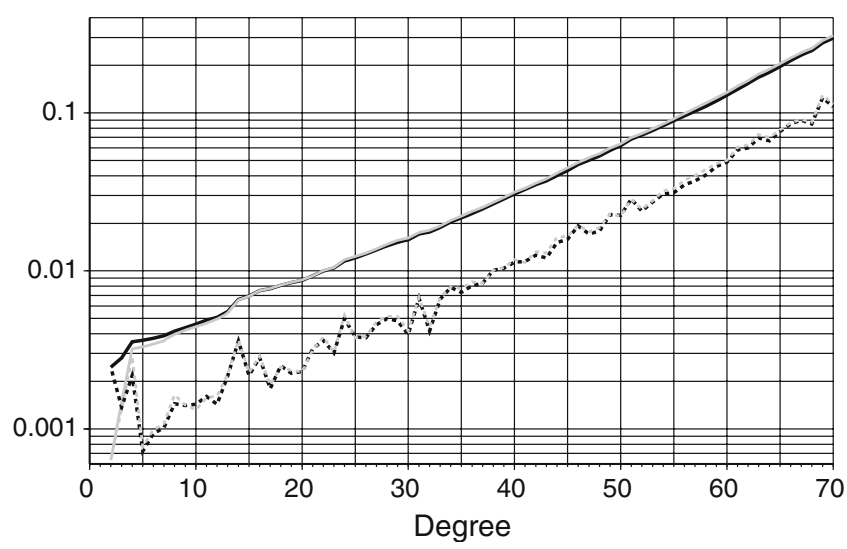

Fig. 9 Accuracy of gravity field models produced from CHAMP accelerations that are cleaned (gray lines) and not cleaned (black lines) from non-gravitational accelerations, in terms of cumulative geoid height errors (solid lines) and geoid height errors per degree (dashed lines). Both gravity field models are obtained with the exact numerical noise models; both the cross-component correlations and the regularization are switched off

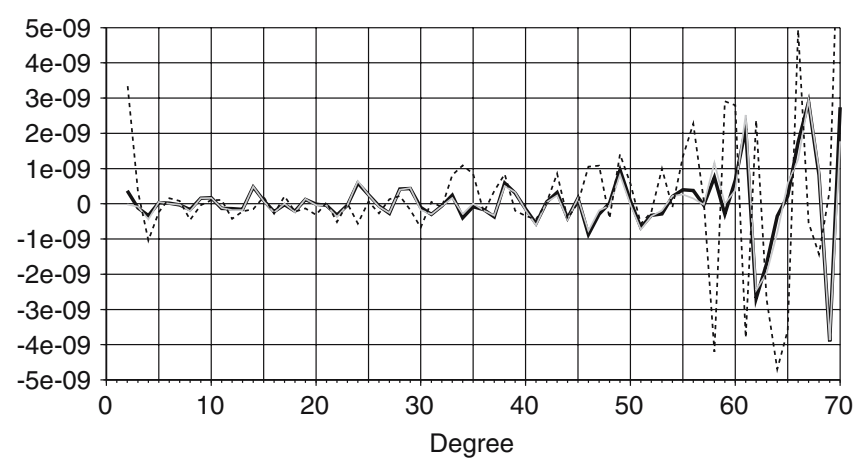

Fig. 10 Accuracy of gravity field models produced from CHAMP accelerations that are cleaned (solid gray line) and not cleaned (solid black line) from non-gravitational accelerations: errors in the zonal coefficients are shown. Furthermore the error difference $\delta_{l} \stackrel{\text { def }}{=} 10 \times$ $\left(\left|C_{l, 0}^{\text {(non-cleaned) }}-C_{l, 0}^{\text {(true) }}\right|-\left|C_{l, 0}^{\text {(cleaned) }}-C_{l, 0}^{\text {(true) }}\right|\right)$ is plotted as a dotted line. $\delta_{l}>0$ if the non-cleaned accelerations give a larger error and $\delta_{l}<0$ if the non-cleaned accelerations give a smaller error. Both gravity field models are obtained with the exact numerical noise models; both the cross-component correlations and the regularization are switched off

the data from non-gravitational accelerations does not lead to a noticeable improvement of the gravity field model above degree 2.

\section{Discussion and conclusions}

We have shown how to handle correlated noise in data, even if a data series contains interruptions. Furthermore, a variable data accuracy can be taken into account provided that the data are supplied with error estimates. The noise model is built up numerically. Therefore, any dependence of noise on frequency can be considered.

To illustrate the proposed methodology, we have shown how it can be exploited in CHAMP data processing. In particular, we have compared the proposed methodology with the one presented earlier, when dependence of noise on frequency was described analytically, by a function with only two adjustable parameters (Ditmar et al. 2004b, 2006b). Though it turned out that both types of noise models perform almost equally, we still believe that the proposed methodology 
is a step forward. Noise in data of other types (in particular, delivered by other satellite missions) can depend on frequency in a completely different way, and the analytic model will be unable to approximate it properly.

A number of interesting aspects were left beyond the scope of the discussion in order to make it better focused. Let us briefly summarize the results that are not included into the main text.

1. Extension of the covariance matrix. We mentioned that the matrix $\mathbf{C}^{(\text {nst) }}$ has to be extended in order to be used in the context of the correlated noise. The new elements of the matrix $\mathbf{C}^{(\mathrm{nst})}$ are set equal to unity. Of course, this is an arbitrary choice. Then, the question arises whether the gravity field model is robust with respect to this choice. Numerical experiments allowed us to give the positive answer. Even if the new elements in the matrix $\mathbf{C}^{\text {(nst) }}$ are defined very differently (e.g. set equal to 100), the change in the resulting gravity field model is negligible.

2. Further improvement of the noise model. In essence, we propose an iterative scheme for estimation of both a noise model and a gravity field model. In the considered example, we stop after two iterations; the corresponding gravity field model is called final. One may think that a final gravity field model can be used for a further improvement of the noise model and, consequently, of the gravity field model. Our experience tells us, however, that the improvement of the noise model after the second iteration is marginal. Therefore, the gravity field model does not improve after two iterations any further. We believe that this observation can be related to the fact that the problems we consider are highly redundant: the number of data is much larger than the number of unknown parameters.

3. Importance of a correlated noise model in general. A question one may pose is whether it makes sense to consider a dependence of noise on frequency at all. Our answer is definitely positive. In general, a simplistic leastsquares adjustment without a proper frequency-dependent data weighting may deteriorate the resulting gravity field model dramatically (Klees et al. 2003; Ditmar and van Eck van der Sluijs 2004). This is particularly true when noise at different frequencies changes by the orders of magnitude. On the other hand, it is important to remember about a link between the magnitude of noise variations and the data sampling rate. For example, satellite accelerations derived from an orbit with 1-s sampling may show a strong dependence of noise on frequency, so that a frequency-dependent data weighting is essential. At the same time, the accelerations derived from an orbit with a 30-s sampling may show a much weaker dependence on frequency, so that reasonable results can even be obtained without a frequency-dependent data weighting (Ditmar et al. 2006b).

It is important to notice that an accurate noise stochastic model may solve the problem of the optimal regularization parameter. As soon as the covariance matrix of the reference gravity field model $\mathbf{C}_{\mathbf{m}_{\mathbf{0}}}$ is known, the statistically optimal combined model is given by Eqs. 6 and 7, which do not contain any regularization parameter, i.e., a scaling factor to be applied to the matrix $\mathbf{C}_{\mathbf{m}_{\mathbf{0}}}$. A regularization parameter is only needed if the latter matrix is not known.

Fortunately, this is not the case for the available covariance matrix of the EGM96 model. Our experience tells us that the optimal combined model is obtained when no scaling is applied to this matrix, provided that an accurate noise stochastic model is used (Ditmar et al. 2006a). This is not fully consistent with previous publications, which claimed that the EGM96 covariance matrix has to be significantly down-scaled (e.g.van Loon and Kusche 2005). We believe that this discrepancy can primarily be explained by the fact that no serious attention was paid so far to a proper data weighting (especially, in the frequency domain).

The proposed data processing methodology has also been applied to CHAMP data under the assumption that no knowledge of non-gravitational forces is available. It was not very surprising to see that the harmonics of relatively high degrees could be restored from these data accurately: nongravitational accelerations mostly manifest themselves at low frequencies. It was more surprising that even low-degree harmonics (except for degree 2) are not deteriorated when the data are not cleaned from non-gravitational accelerations. We believe that there are at least two factors that contributed to such an outcome:

1. Not all observational components are contaminated by non-gravitational accelerations equally. In particular, the cross-track component suffers from these accelerations less than the along-track one. Since the cross-track component is the most accurate one and, consequently, gets the largest weight in the data processing, the influence of non-gravitational accelerations is reduced.

2. Even though the atmospheric drag in the along-track component is relatively large, its direction is dependent on whether the satellite ascends or descends. As far as the number of ascending and descending passes over each geographical area is approximately equal, the signals related to non-gravitational accelerations cancel each other.

Thus, we have been able to demonstrate that an accurate gravity field model can be obtained from a satellite orbit even if the satellite is not equipped with an accelerometer. This opens the door for the utilization of non-dedicated satellites for high-quality gravity field modeling. The pre-requisites are (i) a precise knowledge of the satellite orbit (presumably, derived from GPS data); (ii) a low altitude (at least in the perigee); and (iii) an attitude control, which is needed to make a correction for the offset between the GPS antenna and the satellite center of mass.

The aim of gravity field modeling on the basis of nondedicated missions can be to monitor temporal variations of the gravity field. Even though this task is being solved by the GRACE mission at this moment, it is unwise to ignore possible alternatives. This is because (i) the spatial/temporal resolution of the GRACE mission is limited, and any additional information can improve the resolution (this would be 
important, in particular, for a further improvement of ocean tide models); (ii) the series of GRACE data suffer from interruptions; information delivered by other satellites can, to a certain extent, fill in these gaps; (iii) the GRACE mission will be completed in 2009 or 2010, and no final decision regarding a follow-on mission(s) has been made yet.

Naturally, it goes without saying that additional studies are needed in order to assess the possible contribution of non-dedicated satellites to monitoring temporal variations of the Earth's gravity field. Our preliminary estimations show that non-dedicated satellite missions may indeed contribute to such monitoring provided that the positioning accuracy is improved by at least one order of magnitude compared to the CHAMP mission.

Acknowledgements We thank D. Švehla and M. Rothacher (Technical University of Munich, Germany) for providing us with kinematic and reduced-dynamic orbits of the CHAMP satellite. Furthermore, we are deeply thankful to W.-D. Schuh and two anonymous reviewers for high-quality reviews of a manuscript draft; they helped us to improve the manuscript a lot. Most of the computations were done on the SGI Altix 3700 super-computer in the framework of the grant SG-027, which was provided by "Stichting Nationale Computerfaciliteiten" (NCF). The support of NCF is gratefully acknowledged.

\section{Appendix. Basics of the discrete Fourier transform and circulant matrices}

This appendix concisely presents the basic facts about the discrete Fourier transform (DFT) and circulant matrices. Only the statements directly related to the contents of the article are included (for more information see, e.g., Davis 1979; Voevodin and Tyrtyshnikov 1987).

1. Discrete Fourier transform. Let $\mathbf{x}$ be a vector composed of $N$ real numbers and let $\mathbf{s}$ be its discrete spectrum (a vector comprised $N$ complex numbers). Then, the relationship between $\mathbf{x}$ and $\mathbf{s}$ can be written as

$\mathbf{s}=\frac{1}{N} \mathbf{F}^{*} \mathbf{x}$,

where $\mathbf{F}$ is the DFT matrix of size $N \times N$ with entries defined as $\{\mathbf{F}\}_{m q}=\mathrm{e}^{i m q \frac{2 \pi}{N}}$, and $\mathbf{F}^{*}$ is the Hermitian conjugate (transposed complex conjugate) of $\mathbf{F}$ (for the sake of convenience, all the entries in vectors and matrices hereafter are enumerated starting from 0 , not from 1 ). All the elements of the spectrum $\mathbf{s}$ are real (not complex) if the elements of the original vector $\mathbf{x}$ are symmetric with respect to the element number $N / 2$ (i.e., $\{\mathbf{x}\}_{i}=\{\mathbf{x}\}_{N-i}$ ).

2. Inverse discrete Fourier transform. It is easy to show that $\mathbf{F}^{*}$, up to a scaling factor, coincides with the inverse of $\mathbf{F}$ : $\mathbf{F}^{*} \mathbf{F}=\mathbf{F F}^{*}=N \mathbf{I}$. Therefore, application of the matrix F to both parts of Eq. 36 results in the inverse DFT:

$\mathbf{x}=\mathbf{F s}$,

The DFT is a reversible operation: the sequential application of the direct and inverse Fourier transform results in the original vector.
3. Periodicity properties. In the DFT, a periodicity of vectors both in the time domain and in the Fourier domain is assumed. This means that the result of the DFT does not change if the elements of the input vector are re-ordered in time in a cyclic way, i.e., such that the indices of some elements (at the beginning or at the end of the vector) are increased or decreased by N. Similarly, the result of the inverse DFT does not change if the elements of the input vector are re-ordered in the frequency domain in a cyclic way.

4. Circulant matrices. Let $\mathbf{H}$ be a diagonal matrix of size $N \times N$. Then, a matrix $\mathbf{C}$ defined as

$\mathbf{C}=$ FHF $^{*}$

is a circulant matrix, i.e. a matrix where each row is a copy of the previous one obtained by putting the last element into the first place, so that the whole row gets shifted by one position to the right. Obviously, the whole content of a circulant matrix is defined by the contents of the first row. Application of a circulant matrix to a vector is a cyclic convolution, i.e., the convolution carried out under the assumption that the input and the output vectors are periodic.

5. Circulant matrices and Fourier transforms. Let us introduce a vector $\mathbf{h}$ composed of the diagonal elements of the matrix $\mathbf{H}$ from Eq. 38. Furthermore, let $\mathbf{c}$ be a vector coinciding with the first row of the matrix $\mathbf{C}$. Then, $\mathbf{c}$ is the result of the inverse DFT applied to the vector $\mathbf{h}$ : $\mathbf{c}=\mathbf{F h}$.

6. Filtering. Filtering in the Fourier domain is multiplication of a spectrum with certain filter coefficients: $s_{j}^{(h)}=$ $N h_{j} s_{j}$, where $s_{j}$ are the elements of the input spectrum, $s_{j}^{(h)}$ are the elements of the filtered spectrum, and $N h_{j}$ are the elements of the filter. In matrix-vector notation, this can be written as

$\mathbf{s}^{(\mathbf{h})}=N \mathbf{H s}$.

Application of the inverse DFT to Eq. 39 yields

$\mathbf{x}^{(\mathbf{h})}=N \mathbf{F s}{ }^{(\mathbf{h})}=\mathbf{F H F}^{*} \mathbf{F s}=\mathbf{C x}$,

where $\mathbf{x}$ is the original vector in the time domain, $\mathbf{x}^{(\mathbf{h})}$ is the filtered vector in the time domain, and $\mathbf{C}$ is a circulant matrix defined according to Eq. 38. Thus, filtering in the Fourier domain is equivalent to the cyclic convolution in the time domain.

7. Square root of a circulant matrix. If $\mathbf{C}$ is a circulant matrix defined according to Eq. 38, then

$\mathbf{C}^{\frac{1}{2}}=\frac{1}{\sqrt{N}} \mathbf{F H}^{\frac{1}{2}} \mathbf{F}^{*}$.

Indeed,

$$
\begin{aligned}
\mathbf{C}^{\frac{1}{2}} \mathbf{C}^{\frac{1}{2}} & =\frac{1}{N} \mathbf{F H}^{\frac{1}{2}} \mathbf{F}^{*} \mathbf{F H}^{\frac{1}{2}} \mathbf{F}^{*} \\
& =\mathbf{F} \mathbf{H}^{\frac{1}{2}} \mathbf{H}^{\frac{1}{2}} \mathbf{F}^{*}=\mathbf{F} \mathbf{H F}^{*}=\mathbf{C} .
\end{aligned}
$$

The pre-requisite is that all the diagonal elements of the matrix $\mathbf{H}$ are non-negative. It follows from Eq. 41 that the 
matrix $\mathbf{C}^{\frac{1}{2}}$ is also circulant. Equation 41 can be implemented with ease because $\mathbf{H}$ is a diagonal matrix.

8. Inverse of a circulant matrix. For a circulant matrix $\mathbf{C}$ defined according to Eq. 38, it holds that

$\mathbf{C}^{-1}=\frac{1}{N^{2}} \mathbf{F H}^{-1} \mathbf{F}^{*}$.

Indeed,

$$
\begin{aligned}
\mathbf{C}^{-1} \mathbf{C} & =\frac{1}{N^{2}} \mathbf{F H}^{-1} \mathbf{F}^{*} \mathbf{F} \mathbf{H} \mathbf{F}^{*} \\
& =\frac{1}{N} \mathbf{F H}^{-1} \mathbf{H F}^{*}=\frac{1}{N} \mathbf{F F}^{*}=\mathbf{I} .
\end{aligned}
$$

The pre-requisite is that all the diagonal elements of the matrix $\mathbf{H}$ are non-zero. The matrix $\mathbf{C}^{-1}$ is also circulant. Equation 43 can be implemented with ease.

\section{References}

Bouman J (2000) Quality assessment of satellite-based global gravity field models, Ph.D thesis, Delft University of Technology

Davis PJ (1979) Circulant matrices. Wiley, New York

Ditmar P, van Eck van der Sluijs AA (2004) A technique for Earth's gravity field modeling on the basis of satellite accelerations. J Geod 78:12-33

Ditmar P, Kusche J, Klees R (2003) Computation of spherical harmonic coefficients from gravity gradiometry data to be acquired by the GOCE satellite: regularization issues. J Geod 77:465-477

Ditmar P, Klees R, Kuznetsov V, van Eck van der Sluijs AA, Schrama E, Liu X (2004a) Gravity field analysis with the acceleration approach on the basis of data from the CHAMP satellite mission. In: Proceedings of the IAG international symposium "Gravity, Geoid and Space Missions - GGSM2004", Porto, Portugal, 30 August-3 September, 2004, CDROM

Ditmar P, van Eck van der Sluijs AA, Kuznetsov V (2004b) Modeling the Earth's gravity field from precise satellite orbit data: the acceleration approach works! (available as http://earth.esa.int/workshops/goce04/participants/81/paper_accelerations.p df). In: Proceedings of the 2nd international GOCE user workshop, Frascati (Italy), 8-10 March, 2004. European Space Agency

Ditmar P, Kuznetsov V, van Eck van der Sluijs AA, Klees R (2006a) Modeling of the Earth's gravity field from CHAMP satellite data by means of the acceleration approach. In: Proceedings of the joint CHAMP/GRACE science meeting, GeoForschungsZentrum, Potsdam, 6-8 July, 2004 (submitted)

Ditmar P, Kuznetsov V, van Eck van der Sluijs AA, Schrama E, Klees R (2006b) 'DEOS_CHAMP-01C 70': a model of the Earth's gravity field computed from accelerations of the CHAMP satellite. J Geod 79:586-601

ESA (1999) Gravity field and steady-state ocean circulation missions. Reports for mission selection. The four candidate Earth explorer core missions, SP-1233(1). European Space Agency, Noordwijk

Förste C, Flechtner F, Schmidt R, Meyer U, Stubenvoll R, Barthelmes F, König R, Neumayer KH, Rothacher M, Reigber C, Biancale R, Bruinsma S, Lemoine J-M, Raimondo JC (2005) A new high resolution global gravity field model derived from combination of GRACE and CHAMP mission and altimetry/gravimetry surface gravity data. Poster presented at EGU General Assembly 2005, Vienna, Austria, 24-29 April, 2005. Available as http://www.gfz-potsdam.de/pb1/op/grace/results/grav/g004_EGU05-A-04561.pdf

Hestenes MR, Stiefel E (1952) Methods of conjugate gradients for solving linear systems. J Res Nat Bureau Stand 49:409-436
Kay SM, Marple SL (1981) Spectrum analysis - a modern perspective. Proc IEEE 69(11):1380-1419

Klees R, Broersen P (2002) How to handle colored noise in large leastsquares problems. Building the optimal filter. Delft University Press, Delft

Klees R, Ditmar P (2004) How to handle colored noise in large leastsquares problems in the presence of data gaps? In: Sansò F (ed) V Hotine-Marussi symposium on mathematical geodesy. International association of geodesy symposia, vol 127. Springer, Berlin Heidelberg New York, pp 39-48

Klees R, Ditmar P, Broersen P (2003) How to handle colored observation noise in large-scale least-squares problems. J Geod 76:629-640

Koch K-R, Kusche J (2002) Regularization of geopotential determination from satellite data by variance components. J Geod 76:259-268

Kusche J, Klees R (2002) Regularization of gravity field estimation from satellite gravity gradients. J Geod 76:359-368

Lemoine FG, Kenyon SC, Factor JK, Trimmer RG, Pavlis NK, Chinn DS, Cox CM, Klosko SM, Luthcke SB, Torrence MH, Wang YM, Williamson RG, Pavlis EC, Rapp RH, Olson TR (1998) The development of the joint NASA GSFC and the National Imagery and Mapping Agency (NIMA) geopotential model EGM96. NASA/TP-1998206861. NASA GSFC, Greenbelt, Maryland

Liu X, Ditmar P, Klees R (2006) Estimation of data noise in global gravity field modeling. In: Proceedings of the "Dynamic Planet - 2005" scientific meeting, Cairns, Australia, 22-26 August, 2005 (submitted)

Mayer-Gürr T, Ilk KH, Eicker A, Feuchtinger M (2005) ITGCHAMP01: a CHAMP gravitiy field model from short kinematical arcs of a one-year observation period. J Geod 78:462-480

Migliaccio F, Reguzzoni M, Sansò F (2004) Space-wise approach to satellite gravity field determination in the presence of colored noise. J Geod 78:304-313

Reigber C (1989) Gravity field recovery from satellite tracking data. In: Sansò F, Rummel R (eds) Theory of satellite geodesy and gravity field determination. Lecture Notes on Earth Sciences Vol 25. Springer, Berlin Heidelberg New York, 197-234

Reigber C, Bock R, Forste C, Grunwaldt L, Jakowski N, Lühr H, Schwintzer P, Tilgner C (1996) CHAMP phase B executive summary. GeoForschungsZentrum, STR96/13, Potsdam

Reigber C, Jochmann H, Wünsch J, Petrovic S, Schwintzer P, Barthelmes F, Neumayer K-H, König R, Förste C, Balmino G, Biancale R, Lemoine J-M, Loyer S, Perosanz F (2005a) Earth gravity field and seasonal variability from CHAMP. In: Reigber C, Lühr H, Schwintzer P, Wickert J (eds) Earth observation with CHAMP - results from three years in orbit. Springer, Berlin Heidelberg New York, pp 25-30

Reigber C, Schmidt R, Flechtner F, König R, Meyer U, Neumayer K-H, Schwintzer P, Zhu SY (2005b) An earth gravity field model complete to degree and order 150 from GRACE: EIGEN-GRACE02S. J Geodyn 39:1-10

Reubelt T, Austen G, Grafarend EW (2003) Harmonic analysis of the Earth's gravitational field by means of semi-continuous ephemerides of a low Earth orbiting GPS-tracked satellite. Case study: CHAMP. J Geod 77:257-278

Schuh WD (1996) Tailored numerical solution strategies for the global determination of the Earth's gravity field. Mitteilungen der geodätischen Institute der Technischen Universität Graz. Folge 81. Graz

Schuh WD (2003) The processing of band-limited measurements: Filtering techniques in the least squares context and in the presence of data gaps. Space Sci Rev 108:67-78

Sneeuw NJ (2002) Validation of fast pre-mission error analysis of the GOCE gradiometry mission by a full gravity field recovery simulation. J Geodyn 33:43-52

Švehla D, Rothacher M (2003) Kinematic precise orbit determination for gravity field determination (available as http://tau.fesg.tu-muenchen.de/d̃razen/IUGG03_Svehla.pdf). In: Sansò F (ed) A window on the future of geodesy. IUGG General Assembly 2003, 30 June11 July 2003, Sapporo, Japan. International Association of Geodesy Symposia, vol 128. Springer, Berlin Heidelberg New York, pp 181188 
Tapley BD (1997) The gravity recovery and climate experiment (GRACE). Suppl EOS Trans Am Geophys Union 78(46):163

Tapley B, Ries J, Bettadpur S, Chambers D, Cheng M, Condi F, Gunter B, Kang Z, Nagel P, Pastor R, Poole S, Wang F (2005) GGM02 - an improved Earth gravity field model from GRACE. J Geod 79:467478

Tapley BD, Bettadpur S, Ries JC, Thompson PF, Watkins MM (2004) GRACE measurements of mass variablity in the Earth system. Science 294:2342-2345

Teunissen PJG (2000) Adjustment theory; an introduction. Delft University Press, Delft

Tikhonov AN, Arsenin VY (1977) Solutions of ill-posed problems. V.H. Winston and Sons, Washington

van Loon JP, Kusche J (2005) Stochastic model validation of satellite gravity data: a test with CHAMP pseudo-observations. In: Jekeli C, Bastos L, Fernandes J (eds) Gravity, geoid and space missions. International association of geodesy symposia, vol 129. Springer, Berlin Heidelberg New York, pp 24-29
Voevodin VV, Tyrtyshnikov EE (1987) Vychislitel'nye processy s Toeplitzevymi matritsami (computations with Toeplitz matrices, in Russian). Nauka, Moscow

Wahr J, Swenson S, Zlotnicki V, Velicogna I (2004) Time-variable gravity from GRACE: first results. Geophys Res Lett 31, L11501. doi: 10.1029/2004GL019779

Wermuth M, Švehla D, Földvary L, Gerlach C, Gruber T, Frommknecht B, Peters T, Rothacher M, Rummel R, Steigenberger P (2004) A gravity field model from two years of CHAMP kinematic orbit using the energy balance approach. Oral presentation at the EGU 1st General Assembly, Nice, France, 25-30 April 2004 